\title{
COVID-19 - mehr Lungen-PoCUS und sparsam mit Stethoskop, Thoraxröntgen und Lungen-CT umgehen
}

\author{
COVID-19 - More Lung Pocus and Sparing Use \\ of Stethoscope, Chest X-Ray and Lung CT
}

Joseph Osterwalder

Polipraxis Permanence, St. Gallen

\begin{abstract}
Zusammenfassung: Für ein optimales Management der COVID-19 (Coronary Virus Disease 19) sind wir auf eine schnelle und zuverlässige Diagnose sowie Schweregradeinschätzung angewiesen. Der Goldstandard ist bisher die RT-PCR (Reverse-Transkriptase-Polymerase-Kettenreaktion) aus dem nasopharyngealen Abstrich. Die aktuellen Tests weisen eine Sensitivität zwischen 60 und $90 \%$ auf. In der Folge müssen wir mit 10-40\% falsch-negativen Ergebnissen rechnen. Neben der Sauerstoffsättigung zur Schweregradeinteilung werden das Stethoskop, das Thoraxröntgen und die Lungen-Computertomografie routinemässig für die Diagnose eingesetzt. Die Standardmethoden Stethoskop und Thoraxröntgen sind jedoch unzuverlässig. Überdies setzen alle drei diagnostischen Untersuchungsverfahren Ärztinnen, Ärzte, Hilfspersonal sowie Patientinnen und Patienten einem zusätzlichen Expositionsrisiko aus. Angesichts der Kontagiosität des SARS-CoV-2 (Severe Acute Respiratory Syndrome Corona Virus) ist der Lungen-Point-of-Care-Ultraschall (PoCUS), insbesondere in der Anwendung als Taschenkittelgerät, eine noch zu wenig genutzte, wertvolle Alternative. In dieser Review werden die aktuelle Wertigkeit und Rolle von Stethoskop, Pulsoximetrie, Thoraxröntgen, Lungen-Computertomografie und Lungen-Point-of-Care-Ultraschall anhand der vorliegenden Literatur bestimmt.
\end{abstract}

Schlüsselwörter: COVID-19, bildgebende Verfahren, Lungenultraschall, PoCUS

Abstract: For an optimal management of COVID-19 (Coronary Virus Disease) we depend on a fast and reliable diagnosis and severity assessment. The gold standard so far is RT-PCR (reverse transcriptase polmerase chain reaction) from the nasopharyngeal smear. Current tests have a sensitivity of 60-90\%. As a consequence, we must expect $10-40 \%$ false negative results. In addition to oxygen saturation for severity classification, stethoscope, chest X-ray and lung computer tomography are routinely used. However, the standard methods stethoscope and chest X-ray are unreliable. Moreover, all three diagnostic examination techniques expose physicians, support staff and subsequent patients to an additional risk of exposure. In view of the contagiousness of SARS-CoV-2 (Severe Acute Respiratory Syndrome Corona Virus), lung point-of-care ultrasound (Lu-PoCUS) is a still underutilized valuable alternative, especially when using pocket devices. In this review the current value and role of stethoscope, pulsoxymetry, chest $x$ ray, lung computer tomography and lung point-of-care ultrasound will be determined based on the available literature.

Keywords: COVID-19, imaging, lung ultrasound, PoCUS

Résumé: Pour une gestion optimale du COVID-19 (Coronary Viral Disease), nous dépendons d'un diagnostic rapide et fiable et d'une évaluation de la gravité. L'étalon-or jusqu'à présent est la RT-PCR (Reverse Transcriptase Polymerase Chain Reaction) du frottis nasopharyngien. Les tests actuels ont une sensibilité entre 60 et $90 \%$. En conséquence, on doit compter avec 10 à $40 \%$ de faux résultats négatifs. En plus de la saturation en oxygène pour la classification de la gravité, le stéthoscope, la radiographie pulmonaire et le scanner des poumons (SP) sont couramment utilisés. Cependant, les méthodes standard par stéthoscope et radiographie pulmonaire ne sont pas fiables. De plus, les trois méthodes d'examen diagnostiques exposent les médecins, le personnel auxiliaire et les patients suivants à un risque d'exposition supplémentaire. Compte tenu de la contagiosité du SARS-CoV-2 (Severe Acute Respiratory Syndrome-Corona Virus), l'échographie pulmonaire ciblée (EPC) est une alternative précieuse et encore sous-utilisée, surtout en utilisant des appareils de poche. Dans cette revue, la valeur et le rôle actuels des differents examens stéthosope, pulsoxymétrie, radiographie pulmonaire, scanner des poumons et EPC seront etudés sur la base de la littérature disponible.

Mot-clés: COVID-19, procédés d'imagerie, échographie ciblé, PoCUS 
COVID-19 (Corona Virus Disease 2019) ist eine virale Erkrankung mit bevorzugtem Befall der Atemwege. Es wird aber auch eine direkte Schädigung des zentralen Nervensystems und der Gefässe vermutet. Alles deutet darauf hin, dass die Infektiosität, Morbidität und Mortalität höher sind als bei der saisonalen Grippe. Gemäss aktuellem Erkenntnisstand soll die Übertragung des Virus (SARS-CoV-2) hauptsächlich durch Tröpfchen erfolgen. Diskutiert werden auch Infektionen durch kontaminierte Flächen, Aerosole (die insbesondere bei medizinischen Behandlungen wie zum Beispiel Sauerstoffgabe, Inhalationen und nicht-invasive Ventilation entstehen), Blut sowie auf feco-oralem Weg. Ein nicht unerheblicher Teil der symptomatischen Patienten muss hospitalisiert werden, weil es zu einer absteigenden Infektion der unteren Atemwege bis hin $\mathrm{zu}$ schweren Pneumonien und ARDS kommt. Die Diagnose wird mittels RT-PCR auf SARS-CoV-2 aus einem nasopharyngealen Abstrich gestellt. Angesichts limitierter Ressourcen ist es nun wichtig, dass eine optimale Triage mit sparsamer Testung von Abstrichen, Vermeidung unnötiger Hospitalisationen und Zuweisungen auf die Intensivstation bzw. Verhinderung von Komplikationen durch zu restriktive Handhabung erfolgt. Weiter sind wir auf verlässliche klinische Parameter für das Therapiemonitoring angewiesen.

Im klinischen Alltag basieren Diagnose- und Therapie-Entscheidungen von Erkrankungen der Atemwege auf Anamnese, körperlicher Untersuchung mit dem Stethoskop, Sauerstoffsättigung, funktionellen Tests, Labor und bildgebenden Verfahren. Dies gilt auch für COVID-19. Anamnese und klinische Untersuchung sind wohl wegweisend, jedoch wenig spezifisch. Zudem setzt die Auskultation den Untersucher, der dabei engen körperlichen Kontakt zum Kranken hat, einem unnötigen Übertragungsrisiko aus. Dies, weil die Patienten tief einund ausatmen müssen, so Tröpfchen entstehen und dabei nicht selten Hustenattacken auftreten. Funktionelle Tests sind hier selten notwendig. Laboruntersuchungen mit Ausnahme des Abstrichs bringen leider nicht viel. Der Nachweis mittels RT-PCR ist der Goldstandard mit einer aktuellen Sensitivität zwischen 60 und 90\% [1]. Aus diesem Grund werden zwei negative Tests in einem Abstand von 24 Stunden (in Italien drei Tagen) zum Ausschluss gefordert [1]. Weitere Limitationen sind die beschränkte Verfügbarkeit der Tests und die Dauer (in der Schweiz aktuell ein bis zwei Tage), bis das Ergebnis vorliegt [1]. Allenfalls bringen serologische Point-of -CareAngebote bald eine Verbesserung. In der aktuellen Situation bleiben für die Triage, d.h. Diagnostik und Schweregradeinschätzung, im Notfall nur die Sauerstoffsättigung und drei bildgebende Verfahren übrig. Die Bildgebung spielt aber auch eine Rolle im weiteren klinischen Monitoring.

Nachfolgend werden kurz die diagnostische Wertigkeit und Rolle der zur Verfügung stehenden Hilfsmittel Pulsoxymetrie, Thoraxröntgen, Lungencomputertomografie und Lungenultraschall beschrieben:

\section{Pulsoxymetrie}

Die Bedeutung der Pulsoxymetrie liegt weniger in der Diagnostik als vielmehr in der Einschätzung des Schweregrads bei Patientinnen und Patienten mit Verdacht auf COVID-19 oder nachgewiesenem SARS-CoV-2. Die Höhe der Sauerstoffsättigung hilft nämlich einfach und schnell bei der Entscheidung für eine ambulante oder stationäre Versorgung und ob Patientinnen und Patienten am besten auf die Abteilung, die Intermediate Care oder die Intensivstation verlegt werden sollten.

\section{Thoraxröntgen}

Eine besondere Herausforderung in der aktuellen Situation stellt der Aufwand an Hygienemassnahmen dar. Es gilt, alle Patientinnen und Patienten und das Radiologiepersonal vor Übertragungen im Rahmen von konventionellen Röntgenuntersuchungen zu schützen. Insbesondere der Umgang mit Aerosolbildung bei Sauerstoff-Applikation über $21 / \mathrm{min}$ ist problematisch. Die Indikationen für das konventionelle Röntgen müssen daher sehr streng gestellt werden.

\section{Diagnostische Wertigkeit und Rolle}

Aus der noch spärlichen Literatur scheint das AusgangsThoraxröntgenbild eine tiefere Sensitivität mit 69\% aufzuweisen als die initiale RT-PCR mit 91\% [2]. Allerdings gibt es auch Fälle, in denen das Thoraxröntgenbild vor der RT-PCR positiv ist [2]. Die Situation bezüglich Spezifität ist noch unklar [3], aber sie ist wahrscheinlich höher als die Sensitivität. Eine Fallserie aus Hongkong von 64 Patientinnen und Patienten mit Eintrittsthoraxröntgen setzt an erster Stelle der Befunde Lungenkonsolidationen (50\%), gefolgt von Milchglastrübungen in 33\% [2]. Selten komme es zu Pleuraergüssen. Prädilektionsstellen seien die Peripherie (41\%) und basale Zonen (50\%) sowie eine bilaterale Verteilung (50\%). Eine weitere kleinere Fallserie mit 21 Intensivpatienten aus Seattle beschreibt ein anderes Bild mit 52\% bilateralen reticulo-nodulären Verschattungen, $48 \%$ Milchglastrübungen, 29\% Pleuraergüssen, 25\% peribronchialer Dickenzunahme und 29\% fokalen Konsoli-

$\begin{array}{ll}\text { Im Artikel verwendete Abkürzungen } \\ \text { ACR } & \text { American College of Radiology } \\ \text { ARDS } & \text { Acute Respiratory Distress Syndrome } \\ \text { CDC } & \text { Centers for Disease Control } \\ \text { COVID-19 } & \text { Coronary Virus Disease 2019 } \\ \text { CT } & \text { Computertomografie der Lungen } \\ \text { LU PoCUS } & \text { Point-of-Care Ultraschall der Lungen } \\ \text { RT-PCR } & \text { Reverse-Transkriptase-Polymerase-Kettenreaktion } \\ \text { RTX } & \text { Thoraxröntgen } \\ \text { SARS-CoV-2 } & \text { Severe Acute Respiratory Syndrome Corona Virus 2 } \\ \text { ST } & \text { Stethoskop }\end{array}$


dationen [4]. Diese Unterschiede sind allenfalls auf das Geschlecht, Alter, Stadium, Ethnizität oder regionale Viruscharakteristika zurückzuführen. Ein erster Versuch mit künstlicher Intelligenz für die Bildanalyse ist vielversprechend, muss aber erst an einem grösseren Kollektiv untersucht werden [5].

\section{Wertigkeit und Rolle für das klinische Monitoring}

Die Rolle des klinischen Monitorings mittels Thoraxröntgenbild ist noch unklarer als diejenige bei der Diagnostik. Der radiologische Schweregrad-Peak tritt zehn bis zwölf Tage nach Symptombeginn auf [2]. In einer kleinen Kohorte liess sich kein Unterschied in der medianen Dauer der Erholungsphase zwischen den radiologischen Befunden und der RT-PCR nachweisen [2]. Bei asymptomatischen oder minimal symptomatischen Patienten können positive Röntgenbefunde bis 14 Tage nach Quarantäne auftreten [3]. Es ist bekannt, dass radiologische Verschattungen auch bei abgeheilter Pneumonie noch bis zu Wochen dargestellt werden.

\section{Schlussfolgerungen}

- In der Literatur finden sich unterschiedliche Angaben zu Befunden im Thoraxröntgenbild.

- Das Ausgangs-Röntgenbild hat eine tiefe diagnostische Sensitivität und unklare Spezifität.

- Noch offen ist die Rolle des Thoraxröntgenbilds für das klinische Monitoring.

- Die Centers for Disease Control (CDC) und das American College of Radiology (ACR) empfehlen aus fachlichen, infektiologischen und logistischen Gründen die Röntgenuntersuchung der Lungen nicht für die Diagnosestellung COVID-19 [6].

- Anstelle von fixen Röntgenanlagen sollen mobile Röntgenapparate genutzt werden. Deren Oberfläche ist leichter zu reinigen als Räume, und die Patienten müssen nicht tranportiert werden.

- Es wird sich weisen, ob die Schlussfolgerungen einer Cochrane Review, dass das Thoraxröngenbild bei leichtbis mittelgradigen Infektionen der unteren Atemwege keinen Einfluss auf das Outcome von Patienten hat, auch für COVID-19 gelten [7].

\section{Computertomografie (CT)}

Die Hygienemassnahmen im CT-Untersuchungsraum sind noch aufwendiger als diejenigen bei konventionellen Röntgenanlagen. Auf Details dazu geht ein Erfahrungsbericht aus China ein [8]. Zudem lassen sich respiratorisch insuffiziente und nicht intubierte Patientinnen und Patienten in der CT kaum flach lagern.

\section{Diagnostische Wertigkeit und Rolle}

Die Sensitivität der CT liegt zwischen 91 und 97\% [2, 9]. Andere Autoren sind weniger optimistisch [10]. Es gibt aber auch Hinweise, dass die CT die COVID-19 früher erkennen kann als die RT-PCR [9]. Die CT-Befunde von COVID-19-Patienten sind allerdings wenig spezifisch. So sieht man zum Beispiel ähnliche Bilder bei Fällen mit Influenza. Die Sensitivität und Spezifität von sieben Radiologen in der Unterscheidung von COVID-19 versus NonCOVID-19-Pneumonien liegt zwischen 72 und 94\% bzw. $24 \%$ (ein Ausreisser), sonst zwischen 94 und 100\% [11]. Die wichtigsten Unterscheidungsmerkmale sind eine periphere Anordnung (80 vs 57\%), Milchglastrübung (91 vs $68 \%$ ) und Gefässverdickung (58 vs $22 \%$ ). Optimistisch stimmen die Ergebnisse einer Radiologengruppe aus China [12]. Sie konnten zeigen, dass künstliche Intelligenz in der Lage ist, schnell zu lernen und COVID-19-Pneumonien von «Community-Acquired Pneumonias» sowie anderen Lungenkrankheiten zu unterscheiden (AUC 0,95).

Nun zu den CT-Befunden. Es steht bereits ein systematischer Review mit 919 Patientinnen und Patienten zur Verfügung [13]. Darin werden Milchglastrübungen in $88 \%$ und Konsolidationen in $32 \%$ beschrieben. Bei $88 \%$ der Fälle lag eine bilaterale Beteiligung und bei $79 \%$ eine multilobuläre Verteilung vor. $80 \%$ waren posterior und $76 \%$ peripher gelegen. An weiteren Befunden werden interlobuläre septische Verdichtungen, Bronchiektasen und subpleurale Veränderungen erwähnt. Selten kommen Pleuraerguss, Perikarderguss, Lymphadenopathie, Kavitationen, CT-Halo-Zeichen und Pneumothorax vor.

Akute Infektionen haben ein transient erhöhtes Risiko für Thromboembolien. So wurde die Influenza-Erkrankung als entsprechender Risikofaktor beschrieben [14]. Diese Aussage dürfte auch für COVID-19 gelten [15]. Nicht veröffentlichte Erfahrungen und erste Fallbeschreibungen zeigen in diese Richtung $[16,17]$. Damit ist nicht nur die Differenzialdiagnose Covid-19-Pneumonie versus Lungenembolie eine Herausforderung, sondern auch die Möglichkeit, dass beide gleichzeitig vorhanden sind. Inwieweit in dieser Situation das D-Dimer hilft, ist noch unklar. Bei abnormal erhöhten D-Dimeren, aber auch bei einem plötzlichen Sättigungsabfall sollten wir zumindest daran denken [18]. Als Goldstandard für die Lungenembolie gilt die CT. Auf die wichtige Rolle des LUS werden wir weiter unten eingehen. Weiter wissen wir, dass erhöhte D-Dimere mit einem schlechteren Outcome assoziiert sind.

\section{Wertigkeit und Rolle fürs klinische Monitoring:}

Eine chinesische Gruppe unterscheidet fünf zeitliche Phasen: 1. Ultrafrüh - asymptomatisch und eine bis zwei Wochen nach Exposition; 2. Früh - frühe symptomatische Fälle (54\%); 3. Schnelle Progression - drei bis sieben Tage symptomatisch; 4. Konsolidation - 2. Woche; 5. Auflösung zwei bis drei Wochen nach Beginn [19]. 
Derzeit fehlen jedoch Studien, welche den Einfluss von Verlaufs-CT- Befunden auf das klinische Management untersucht haben.

Akute Lungenembolien sind auch mit ein Grund für die klinische Verschlechterung von viralen Pneumonien, mit anderen Worten müssen wir nicht nur an sekundäre bakterielle Superinfekte denken, sondern auch an Lungenembolien und kardiale Komplikationen, wenn Patienten im Verlauf respiratorisch dekompensieren, die Sättigung fällt oder Symptome wie Tachykardie, neu pleuritische Schmerzen usw. auftreten. Es scheint, dass sich bei COVID-19-Lungenembolien erst im späteren Verlauf der Erkrankung oder in der Erholungsphase (cave nicht nur Rückfälle) bilden.

\section{Schlussfolgerungen}

- Die CT ist dem konventionellen Thoraxröntgen überlegen [2].

- Aus infektiologischen (Infektionsrisiko), medizinischen (schwierig für respiratorisch und hämodynamisch instabile Patienten) und logistischen (zeit- und personalintensiv) Gründen ist die CT-Untersuchung bei COVID-19-Patienten ungeeignet für die Triage und das klinische Monitoring. Trotzdem wird die Lowdose-CT der Lungen in der Schweiz häufig als Screening-Methode verwendet (persönliche Mitteilung von Kollegen).

- Das American College of Radiology empfiehlt [6]: 1. Die CT sollte nicht als Screening oder Erstlinientest für die Diagnose COVID-19 verwendet werden. 2. Die CT sollte restriktiv und nur für hospitalisierte, symptomatische Patienten mit spezifischen Indikationen verwendet werden. 3. Adäquate Massnahmen zur Infek-

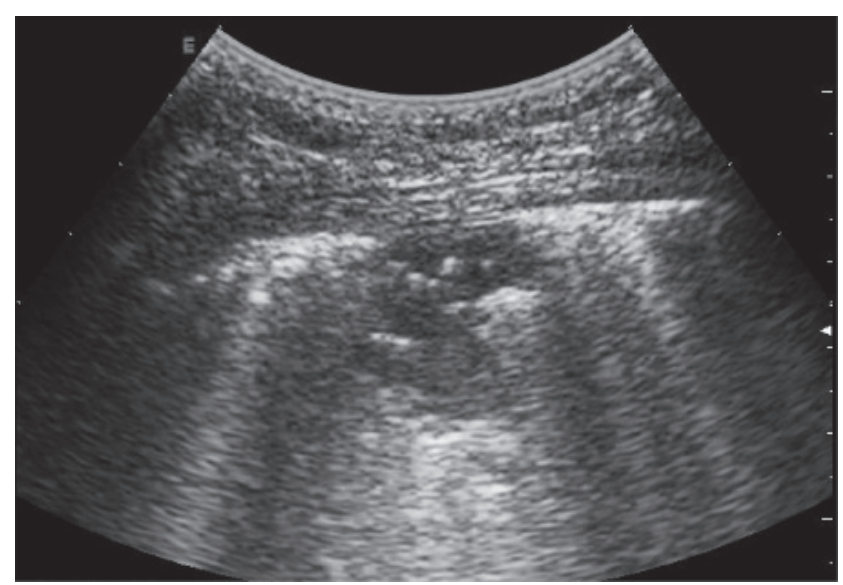

Abbildung 1. H1N1-Pneumonie einer 27-jährigen Patientin mit leichtem inspiratorischem Schmerz, etwas Husten und Fieber $38,5^{\circ} \mathrm{C}$. Unregelmässige Pleuralinie mit Kometenschweifartefakten und kleiner subpleuraler Konsolidierung mit zackiger, unscharfer Begrenzung und Airbronchogramm sowie vom Rand ausgehenden Kometenschweifartefakten (mit freundlicher Genehmigung von Prof. Gebhard Mathis). tionsvorbeugung müssen vor und währed der nächsten Untersuchung eines Patienten vorgenommen werden

- Die Low-dose-CT verringert wohl die Strahlenexposition, das erhöhte Infektionsrisiko jedoch bleibt. Zudem fehlen entsprechende Studien für COVID-19.

- Eine spezielle Indikation für die CT (Angio-CT) ist der Ausschluss einer Lungenembolie.

\section{Ultraschall}

Im Vordergrund steht der Lungenultraschall. Weitere Applikationen wie zum Beispiel die fokussierte Echokardiografie bei Verdacht auf Herzbeteiligung und beim Volumenmanagement sowie gegebenenfalls die Venensonografie bei dringendem klinischem Verdacht auf Thrombose dürfen nicht vergessen gehen. Dazu verweisen wir auf die entsprechende Literatur [20].

\section{Lungenultraschall (LUS)}

Im Gegensatz zur CT kann der LUS als Point-of-CareVerfahren direkt am Patientenbett angewendet werden und ist ein etabliertes Verfahren bei bakteriellen Pneumonien. Er hat in dieser schwierigen COVID-19-Krise viele Vorteile: kein Transport des Patienten, sofort verfügbar, Exposition nur einer Person, einfache Desinfektion, kein Personalaufwand, Zeitersparnis, fehlende Strahlenexposition, kostengünstig und beliebig wiederholbar.

Aus der CT-Literatur wissen wir, dass pathologische Befunde von COVID-19-Patienten häufig an der Peripherie vorkommen $[11,13]$. Sie sind damit dem LUS gut zugänglich. Isolierte, rein zentral gelegene, für den LUS nicht zugängliche Veränderungen scheinen eher seltener aufzutreten. Zudem scheint der LUS der CT bei diskreten pleuralen und subpleuralen Befunden überlegen zu sein.

Leider sind Arbeiten über Viruspneumonien im Gegensatz zu bakteriellen noch spärlich. In einer kleinen Fallserie von 16 Patienten anlässlich der Schweinegrippe 2009 wird ein inhomogenes interstitielles Syndrom mit ausgesparten Zonen beschrieben [21]. Es stimmt gut mit den CT-Befunden einer interstitiellen Pneumonie überein und soll sich für die Schweregradeinschätzung eignen. Eine zweite Arbeit bei 20 Patienten unter 21 Jahren bestätigt diese Aussage [22]. Sie weist auf die Möglichkeit einer Differenzierung von viralen gegenüber bakteriellen Pneumonien hin und schreibt dem LUS eine wichtige Rolle bei Pandemien zu. Weitere Fallbeschreibungen zur Vogelgrippe 2013 zeigen ähnliche Bilder [23-25]. Zusammenfassend handelt es sich um folgende sonografische Zeichen: einzelne und zusammenfliessende vertikale Lungenartefakte von der Pleuralinie ausgehend, Pleuraveränderungen inkl. kleine subpleurale Konsolidationen, von deren Rand vertikale Lungenartefakte ausgehen, grössere Konsolidationen, ausgesparte Zonen und Pleuraergüsse (Abb. 1). 


\section{Diagnostische Wertigkeit und Rolle}

Aktuell fehlen Arbeiten zur diagnostischen Wertigkeit des Lungenultraschalls bei COVID-19. Die spärlichen Fallbeschreibungen oder Fallserien erwähnen nur die sonografischen Befunde (Abb. 2, 3): multifokale, abgrenzbare und/ oder zusammenfliessend vertikale Lungenartefakte, verdickte, unregelmässige und/oder fragmentierte Pleuralinie mit und ohne subpleurale Ergüsse von 2-3 mm Ausdehnung, kleine subpleurale Konsolidationen und/oder nichttranslobäre und translobäre Konsolidationen mit und ohne Airbronchogramm in multilobärer Verteilung und häufigster Lokalisation beidseits posterior [26-30]. In einer noch nicht Peer-reviewten Arbeit wird postuliert, dass die CT bei intrapulmonal gelegenen und apikalen Läsionen dem Ultraschall diagnostisch überlegen und bei kleinen peripulmonalen und pleuralen Läsionen sowie peripulmonalen Ergüssen unterlegen sei [30]. Ein Online-Kurzbeitrag aus Italien kommt ohne Angabe von Zahlenmaterial, zum Schluss, dass der LUS die Identifikation von Patienten mit

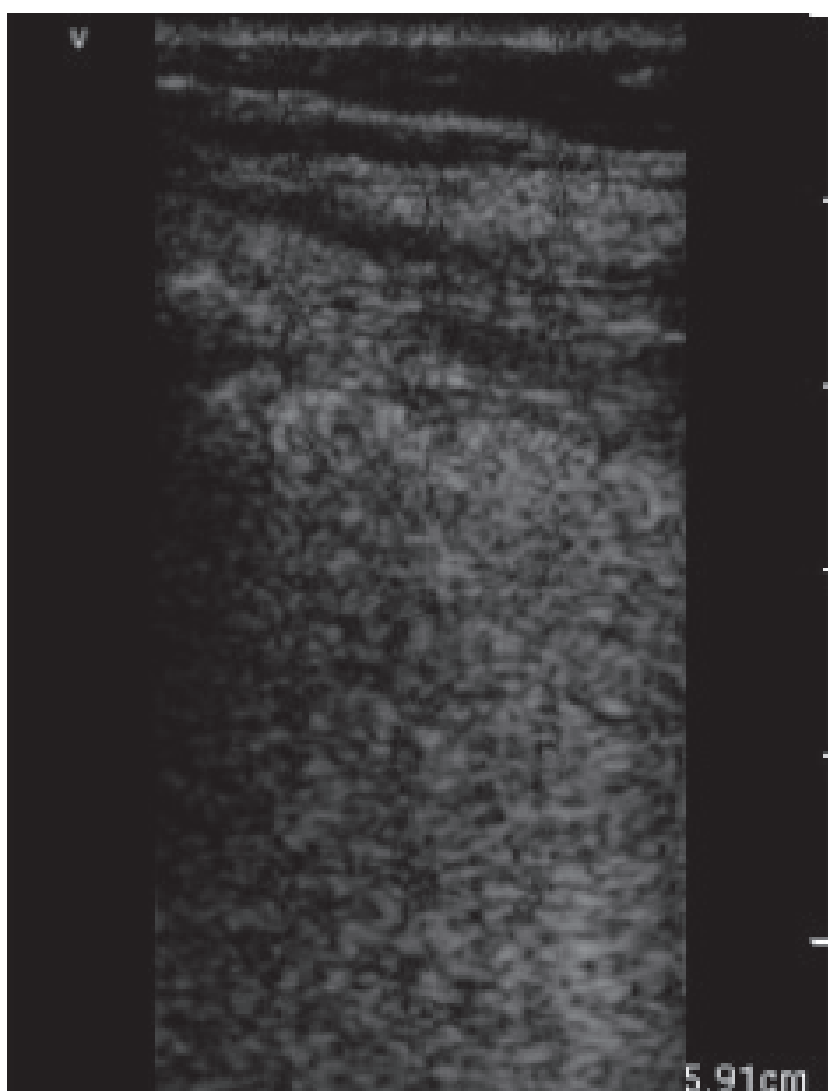

\#18 Lunge

Ml: 0.6 TIs: 0

Abbildung 2. Bild von Taschenkittelgerät: Patient Covid-19-positiv mit irregulärer Pleuralinie und vertikalen Artefakten (mit freundlicher Genehmigung von PD Dr. Konrad Stock).
Lungenbeteiligung und die Einschätzung des Schwergrad erlaubt [31]. Sie postulieren ebenfalls eine Reduktion von Thoraxröntgen- und CT-Untersuchungen dank LUS.

Zur Rolle von LUS bei der Diagnostik gibt es kaum Literatur. Die Gruppe um Gino Soldati, einem Experten auf dem Gebiet der LUS, sieht die Rolle der LUS: 1 . in der Triage zuhause und auf Notfallstationen; 2. in der PrognoseStratifizierung; 3. in der Reduktion des Übertragungsrisikos, d.h. kein Transport und nur eine Person, der Sonografeur, muss geschützt werden [32]. Es kursieren bereits noch nicht validierte Anwendungsprotokolle aus Italien und Deutschland [33, 34]. Die Italiener schlagen 14 Lungenschallfenster pro Seite vor und ein Score-System: Score 0 = normale Pleuralinie und A-Linien; Score 2 = unterbrochene Pleuralinie mit darunter dunklen Konsolidationen, gefolgt von weissen Zonen; Score 3 = dichte und ausgedehnte weisse Lungen mit oder ohne grössere Konsolidationen [33]. Die höchsten Scores aus allen Zonen werden zusammengezählt. Sie fordern alle Kolle-

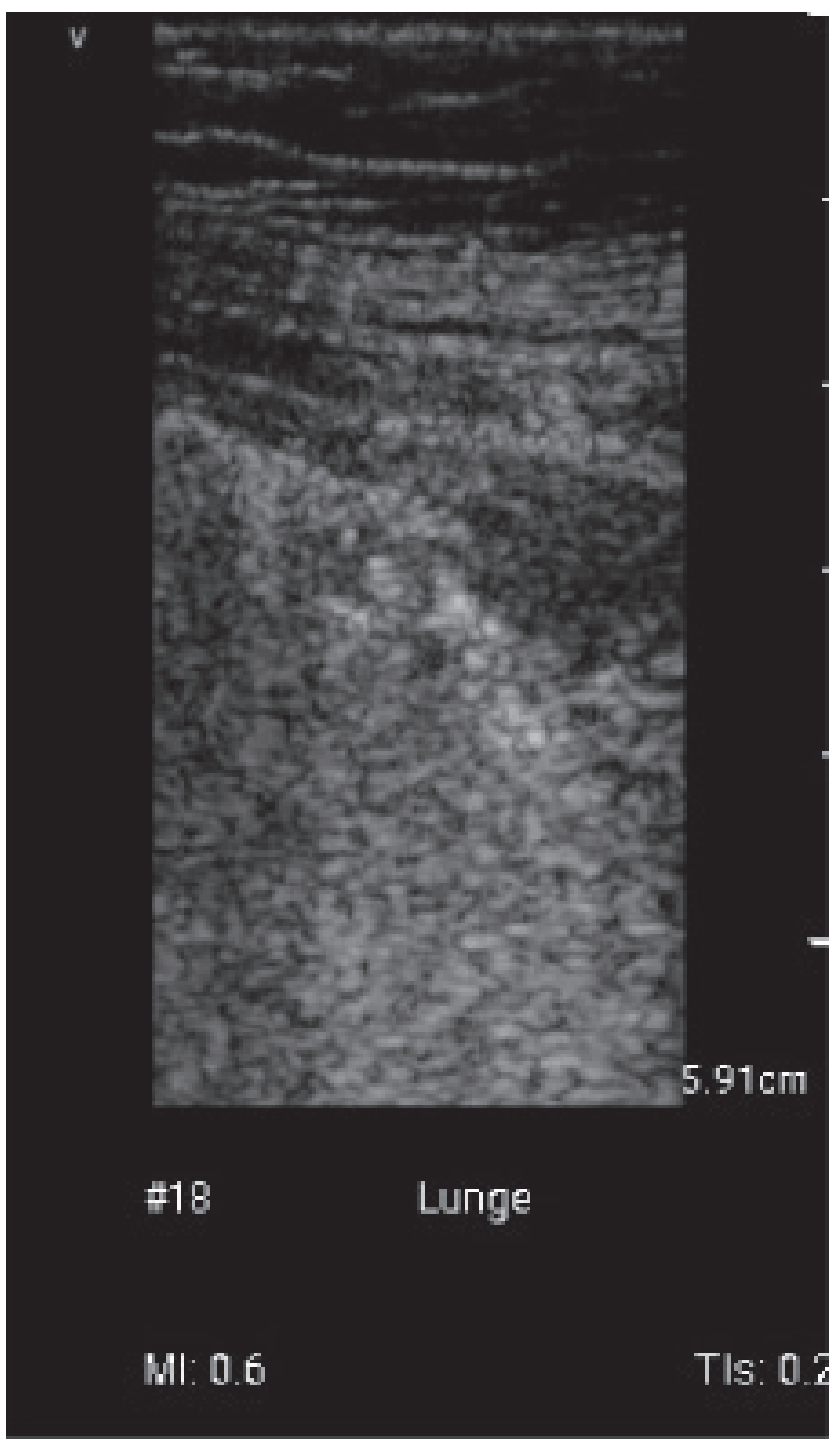

Abbildung 3. Bild von Taschenkittelgerät: Patient Covid-19-positiv mit weisser Lunge (konfluierende vertikale Artefakte) und subpleurale Konsolidation (mit freundlicher Genehmigung von PD Dr. Konrad Stock). 
ginnen und Kollegen auf, ihre Bildgebung (Thoraxröntgen, Ultraschall und CT) auf eine gemeinsame, internationale Datenbank abzulegen mit folgendem Link: https:// covid19.disi.unit.it/iclusdb. Ziel sind die Mustererkennung und Definition der Pathologie, der Vergleich unter den verschiedenen Zentren und die Erarbeitung von Grundlagen für die Entwicklung von telemedizinischen Programmen. Die deutschsprachigen Kollegen beschränken sich auf sechs Schallfenster pro Lungenseite und empfehlen vier Sonokriterien: 1. Pathologische Pleura, 2. Konsolidierungen, 3. Airbronchogramm und 4. B-Linien und Kometenschweifartefakte [34]. Die sechs bzw. zwölf Schallfenster basieren auf den Erfahrungen von Peng et al. [14]. Mike Stone propagiert einen einfachen, vielversprechenden, jedoch nicht validierten klinischen Algorithmus mit vier Möglichkeiten: 1. Kein Sauerstoff und A-Linien - nach Hause in Selbstquarantäne; 2. Kein Sauerstoff und B-Linien - nach Hause in Selbstquarantäne und Follow-up abmachen; 3. Sauerstoffbedürftig und BLinien - Krankenhauseinweisung mit täglichem LUS; 4. Sauerstoffbedürftig und Konsolidationen - IPS-Aufnahme in Betracht ziehen und täglicher LUS [35].

Die Differenzialdiagnose Lungenembolie gehört in die Abklärung von Patienten mit Dyspnoe, Tachykardie, Husten oder Brustschmerzen. Dies gilt auch umso mehr für die COVID-19, wo ein erhebliches Risiko für Thromboembolien besteht. Mehrere Meta-Analysen und eine Konsensuspapier zeigen, dass der LUS, insbesondere kombiniert mit der Echokardiografie und Venenkompressionssonografie, hier eine wichtige Rolle spielen kann [36]. Ein sicherer Ausschluss von Lungenembolien ist damit leider nicht möglich. Dank positiven oder plausiblen alternativen Befunden lassen sich jedoch Angio-CT-Untersuchungen einsparen. Wir sollten mehr zum Ultraschall greifen, da

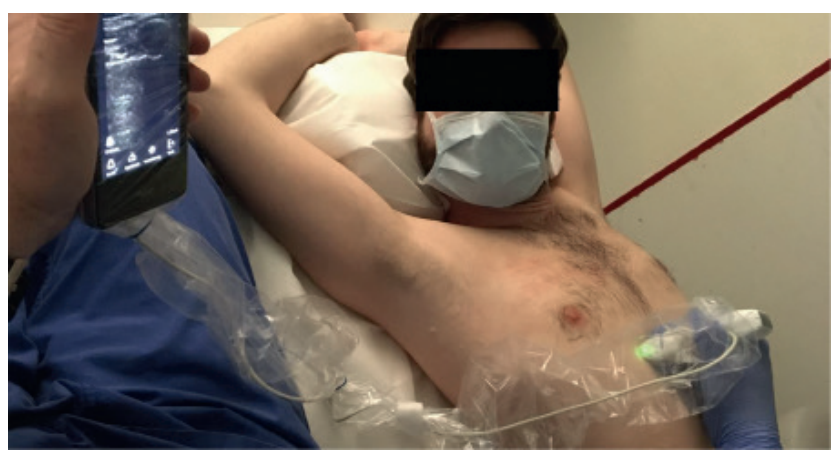

Abbildung 4. Eingepacktes Taschenkittelgerät im Training für Intensivmediziner (mit freundlicher Genehmigung von PD Dr. Konrad Stock).

die Ausbeute von positiven Befunden anlässlich der Angio-CT zwischen 1,3 und 4,8\% liegt [37], d.h. über 95\% der Untersuchungen unnötig waren.

Die Bestimmung der diagnostischen Rolle des LUPoCUS bei COVID-19 basiert auf den wenigen aktuellen Fallberichten/-serien zu COVID-19 und der Extrapolarisation von Befunden aus den spärlichen Ultraschall-Arbeiten über andere Viruspneumonien sowie der ausgedehnteren CT-Literatur betreffend COVID-19 und weiteren Viren.

Schliesslich spielen adäquate Hygienemassnahmen eine entscheidende Rolle [38]. Bei nicht portablen Geräten müssen alle unnötigen Materialien von der Maschine, den Körben und offenen Stauräumen entfernt werden. Man soll Schutzkleidung gemäss Krankenhausrichtlinien tragen. Bei Patienten mit Übertagungsrisiko Tröpfchen: nach Beendigung, Handschuhe desinfizieren und Gel, Körperflüssigkeiten oder andere Verunreinigungen abwischen und desinfizieren. Alle Oberflächen, mit welchen der Patient in Kontakt gekommen ist und welche vom Untersucher berührt worden sind, desinfizieren. Bei Patienten mit Aerosol-

Tabelle 1. Sonografische Differenzialdiagnose.

\begin{tabular}{|c|c|c|c|}
\hline Sonografische Zeichen & COVID-19 & Bakterielle Pneumonie & Lungenembolie \\
\hline Vorkommen & Multifokal oder fleckartig verteilt & In der Regel solitär (fokal) & Multifokale Verteilung \\
\hline Pathologie Pleuralinie* & $\mathrm{Ja}$ & In der Regel nein & In der Regel nein \\
\hline B-Linien oder Kometenschweifartefakte** & $\mathrm{Ja}$ & Am Rand & In der Regel nein \\
\hline Kleine subpleurale Konsolidationen $(<2 \mathrm{~cm})$ & $\mathrm{Ja}$ & Nein & $\mathrm{Ja}$ \\
\hline Grosse Konsolidationen & $\mathrm{Ja}$ & $\mathrm{Ja}$ & Seltener \\
\hline Airbronchogramm*** & $\mathrm{Ja}$ & $\mathrm{Ja}$ & Nein \\
\hline Fluidobronchogramm & $?$ & $\mathrm{Ja}$ & Nein \\
\hline Pleuraerguss**** & $\begin{array}{l}\text { Lokalisert subpleural ja } \\
\text { Basal eher seltener }\end{array}$ & $\begin{array}{l}\text { Komplexer Erguss } \\
\text { (metapneumonisch) }\end{array}$ & Ja (lokalisert und basal) \\
\hline Farbdoppler (CEUS) in Konsolidationen & ? & Vermehrt Farbsignale & Fehlt in der Regel \\
\hline
\end{tabular}

* Die Pleuralinie kann verdickt, unregelmässig, zerhackt und unterbrochen sein mit normalem oder vermindertem bzw. fehlendem Pleuragleiten. ** In dieser Arbeit werden B-Linien und Kometenschweifartefakte folgendermassen unterschieden: Beide sind vertikale, laserstrahlartige Wiederholungsartefakte. B-Linien gehen von einer intakten Pleuralinie aus und Kometenschweifartefakte von einer irregulären Pleuralinie oder vom Rand von Konsolidationen. *** Wir unterscheiden das statische vom dynamischen Airbronchogramm. In der COVID-19-Literatur fehlt diese Differenzierung. Typisch für die Pneumonie ist das dynamische. Beim statischen muss an

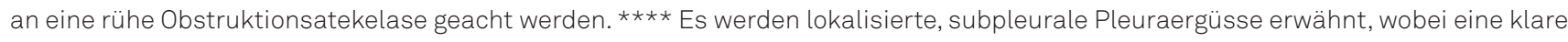
Beschreibung dazu fehlt. 
Übertragungsrisiko: Sonde und Kabel mit spezieller Hülle (Abb. 4) oder zumindest Sonde mit nicht-sterilem Handschuh schützen. Sonde, Keyboard, Bildschirm, Stromkabel, Gehäuse, Räder, Körbe, Gel-Flaschen (allenfalls entsorgen - idealerweise Einmal-Geltüten verwenden) und Stauraum desinfizieren. Unbedingt «Wet-Time» beachten. Die Sonden und das US-Board sollen gemäss Hersteller desinfiziert werden, in der Regel mit 75\% Alkohol, quaternärem Ammoniumsalz oder Hydrogen-Peroxyd.

\section{Wertigkeit und Rolle fürs klinische Monitoring}

Die Gruppe um G. Soldati meint aufgrund noch nicht publizierter Erfahrungen, dass die erste Manifestation einer Covid-19-Pneumonie fleckartig verteilte, einzelne oder zusammenfliessende vertikale Artefakte (bisher «B-Linien» genannt), welche zu echoreichen «weissen» Lungenanteilen führen, seien [32]. Diese verteilen sich an multiplen Stellen der Lungenoberfläche. Im weiteren Verlauf treten immer noch fleckartig verteilte, kleine subpleurale Konsolidationen zusammen mit «weissen» Lungenarealen auf. Beim Übergang in grössere Konsolidationen, speziell der Schwerkraft folgend, mit oder ohne Airbronchogramm und einer zunehmenden Ausbreitung entlang der Lungenoberfläche kündigt sich das Atemversagen an. Pleuraveränderungen und subpleurale Ergüsse werden nicht erwähnt. Dieselben Autoren sehen daher die Rolle von LUS auch im Monitoring von therapeutischen Effekten und für das Management der Beatmung und des «Weanings». Ein deutscher Kollege, der routinemässig die LUS-Befunde von stationär aufgenommen Patienten mit CT-Untersuchungen vergleicht, kann diese Einschätzung nicht teilen. Seiner Meinung nach eignet sich die Ultraschallmethode sehr gut für die Triage, jedoch weder für die Graduierung noch für die Verlaufskontrollen, weil sich die Verschlechterung häufig zentral abspiele.

Schliesslich gilt wie bei der CT-Besprechung oben, dass man bei plötzlicher respiratorischer Verschlechterung an

Tabelle 2. Mögliche Schweregrade

\begin{tabular}{ll}
\hline Stadien & Sonografische Zeichen \\
\hline 0 & - Normale Pleuralinie \\
& - Normales Lungengleiten \\
& - A-Linien \\
& - Reduziertes Lungengleiten \\
- Wenig fragmentierte Pleuralinie & - Einzelne B-Linien oder bei fragmentierter Pleuralinie \\
& Kometenschweifartefakte (max. 2-3 pro sagittalem \\
& Schnittbild zwischen 2 Rippen) \\
& - Stark abnormale Pleuralinie und kleine subpleurale \\
& Konsolidationen \\
& - 4 oder mehr B-Linien oder Kometenschweifartefakte, \\
& welche zum Teil zusammenfliessen \\
& \\
& - Grössere Konsolidationen mit Airbronchogramm \\
& Gegebenenfalls Pleuraerguss
\end{tabular}

\section{Key messages}

- Treu dem hippokratischen Prinzip «Primum non nocere» gilt es, nososokomiale SARS-CoV-2-Infektionen zu minimieren. Mit «mehr Lu-PoCUS und sparsamem Umgang mit Stethoskop, Thoraxröntgen und Lungen-CT» können wir dieses Ziel erreichen und den Eigenschutz für Medizinalpersonen verstärken.

- Keine Schnellbleichen und Experimente! Voraussetzung für den Einsatz von LUS ist eine entsprechende Expertise.

- Die klinische Einschätzung ist die zwingende Basis für jede Bildgebung!

- Thoraxröntgen und CT Thorax werden für die Primärdiagnostik nicht empfohlen (American College of Radiology). Falls in einer gegebenen klinischen Situation trotzdem notwendig, mobile Röntgengeräte benutzen. CT-Untersuchungen (Low-dose-Protokolle verwenden) erfordern spezielle Indikationen, sind auch nicht überall verfügbar und können bei manchen Intensivpatienten mangels Transportfähigkeit nicht eingesetzt werden.

- Obwohl eine sichere Bewertung des LUS für COVID-19 noch fehlt, dürfen wir aufgrund der täglich wachsenden Publikationen darauf schliessen, dass der Pointof-Care-LUS eine zentrale Rolle in der Triage und allenfalls auch für Verlaufskontrollen spielen könnte. Stethoskop nur benutzten, wenn daraus Konsequenzen für das weitere Management erwartet werden und keine Ultraschallmöglichkeiten zur Verfügung stehen.

- Auch in Covidzeiten an kardiale Komplikationen und die Lungenembolie denken und den PoCUS als erste Bildgebung rasch anwenden.

- Offizielle Hygienerichtlinien strikt einhalten: Schutzkleidung! Bestmöglichst Abstand wahren! Auch Gelflaschen in die Planung einbeziehen! Kitteltaschenultraschallgeräte bevorzugt anwenden!

- Sich an ein publiziertes LUS-Untersuchungsprotokolle halten und gut dokumentieren (spätere Auswertung).

- Klinische Pfade und Algorithmen, welche den klinischen Entscheidungsprozess am Patientenbett unterstützen, sind wertvoll und sollen so schnell als möglich entwickelt und validiert werden.

Lungenembolien, welche gehäuft im Verlauf auftreten, denken sollte. Dabei könnte der Ultraschall im Rahmen eines validierten Vorgehens mit Risikostratifizierung helfen, die Anzahl CT-Untersuchungen zu minimieren.

\section{Schlussfolgerungen}

- Die Literatur zu COVID-19-LUS-Literatur ist noch spärlich. Hypothetisch gehen wir jedoch davon aus, dass die Ultraschallerfahrungen mit andern Viruspneumonien/ 
ARDS auch für COVID-19-Pneumonien gelten und dass die vielfach beschriebenen peripheren CT-Veränderungen bei COVID-19-Patienten ebenfalls im Ultraschall ersichtlich sind.

- Weil pathologische Befunde bevorzugt an der Lungenoberfläche vorkommen und der Ultraschall wegen besserer Auflösung der CT überlegen ist, dürfte sich der LU-PoCUS in dieser Situation zumindest für die Triage als ideales bildgebendes Verfahren unter strikter Einhaltung der Hygienemassnahmen erweisen.

- Die sonografischen LUS-Befunde sind unspezifisch. Sie reichen von Pleuraveränderungen über ein spezielles interstitielles Syndrom bis zu grossen Konsoldationen. Lokalisation vorwiegend posterior, bilateral und peripher. Tabelle 1 versucht den aktuellen Stand und die Differenzialdiagnose zur bakteriellen Pneumonie zusammenzufassen. Tabelle 2 gibt einen Überblick über mögliche, bisher nicht validierte und kontrovers beurteilte Stadien. Weitere Studien werden erst zeigen, was daran richtig und was falsch ist.

- Trotz fehlender wissenschaftlicher Untersuchungen und infolge mangelnder guter Alternativen wird der LUS als zentrales Mittel zur Früherkennung und Triage (präklinisch und in den Notfallstationen), mit Vorsicht für die Risiko- und Behandlungsstratifikation sowie das klinische Monitoring (Früherkennung von Komplikationen und Therapie-Effekten, Beatmungskontrolle) und schliesslich für das «Weaning» empfohlen.

- Die Untersuchung mit einem standardisierten Untersuchungsbogen ist empfehlenswert. Zur Verfügung stehen zum Beispiel das DEGUM-, OEGUM-, SGUM Protokoll (einfacher) oder das italienische Protokoll (Vorteil: Sammlung in Datenbank und Schweregradeinteilung; Nachteil: zu kompliziert [33, 34].

- Wir benötigen Algorithmen, damit wir die einzelnen sonografischen Befunde in klinische Entscheidungen übersetzen können.

- PoCUS hat weiter eine grosse Bedeutung für die Diagnostik von Patienten mit bakterieller Pneumonie, kardialen, thromboembolischen und renalen Komplikationen. Insbesondere an die gehäuft vorkommenden Lungenembolien denken und den LUS erweitert um die fokussierte Echokardiografie anwenden.

- Befunde gut dokumentieren, damit später eine wissenschaftliche Auswertung erfolgen kann.

\section{Bibliografie}

1. Giwa AL, Desai A, Duca A: Novel 2019 coronavirus SARS-CoV-2 (COVID-19): An updated overview for emergency clinicians. Emerg Med Pract 2020; 22: 1-28.

2. Wong HYF, Lam HYS, Fong AHT, et al.: Frequency and distribution of chest radiographic findings in COVID-19 positive patients. Radiology 2019; 201160. doi: 10.1148/radiol.2020 201160 (Epub ahead of print).

3. Bandirali M, Sconfienza LM, Serra R, et al.: Letter to the editor: Chest $\mathrm{x}$-ray findings in asymptomatic and minimally symptomatic quarantined patients in Codogno, Italy. Radiology 2020; 201102. doi:10.1148/radiol.2020201102 (Epub ahead of print).

4. Arentz M, Yim E, Klaff L, et al.: Characteristics and outcomes of 21 critically ill patients with CVOVID-19 in Washington State. JAMA 2020; e204326. doi: 10.1001/jama.2020.4326.

5. Hall LO, Paul R, Goldgof DB, Goldgof GM: Finding Covid-19 from chest $x$-rays suing deep learning on a small dataset. arXiv.2004.0260 (eess.IV).

6. ACR recommendations for the use of chest radiography and computed tomography for suspected COVID-19 infection. https://www.acr.org/Advocacy-and-Economics/ACR-PositionStatements/Recommendations-for-Chest-Radiography-andCT-for-Suspected-COVID19-Infection; letzter Zugriff 02.04.2020.

7. Cao AMY, Choy JP, Mohanakrishnan LN, Bain RF, van Driel ML: Chest radiographs for acute lower respiratory tract infections. Cochrane Database Syst Rev. 2013; 2013 (12): CD009119.

8. An P, Ye Y, Chen M, et al.: Management strategy of novel coronavirus (COVID-19) pneumonia in the radiology department: a Chinese experience. Diagn Interv Radiol 2020; 10.5152/dir. 2020.20167 (Epub ahead of print).

9. Ai T, Yang Z, Zhan C, et al.: Correlation of chest CT and RT-PCR testing in Coronavirus Disease 2019 (Covid-19) in China: Report of 1014 cases. Radiology 2020; 200642. doi: 10.1148/ radiol.2020200642 (Epub ahead of print).

10. Inui S, Fujikawa A, Jitsu M, et al.: Chest CT findings in cases from the cruise ship «Diamond Princess» with Coronavirus Disease 2019 (COVID-19). Radiology Cardiothoracic Imaging. 2020 Mar 17 doi: 10.1148/ryct.2020200110 (Epub ahead of print).

11. Bai HX, Hsieh B, Xiong Z, et al.: Performance of radiologists in differentiating COVID-19 from viral pneumonia on chest CT. Radiology 2020; 200823:. 10.1148/radiol.2020200823 (Epub ahead of print).

12. Li L, Qin L, XU Z, et al.: Artificial intelligence distinguishes COVID-19 from community acquired pneumonia on chest CT. Radiology 2020; 200905. doi: 10.1148/radiol.2020200905 (Epub ahead of print).

13. Salehi S, Abedi A, Balakrishnan S, Gholamrezanehad A: Coronavirus disease 2019 (Covid-19): A systematic review of imaging findings in 919 patients. AJR Am J Roentgenol 2020; 1-7. doi: 10.2214/AJR.20.23034 (Epub ahead of print).

14. Armstrong SM, Darwish WI, Lee WL: Endothelial activation and dysfunction in the pathogenesis of influenza A virus infection. Virulence 2013; 4: 537-542.

15. Driggin E, Madhavan MV, Bikdeli B, et al.: Cardiovascular considerations for patients, health care workers, and health systems during the coronavirus disease 2019 (COVID-19) pandemic. J Am Coll Cardiol 2020; S0735-1097(20)34637-4. doi: 10.1016/j.acc.2020.03.031.

16. Danzi GB, Loffi M, Galeazzi G, Gherbesi E: Acute pulmonary embolism and COVID-19 pneumonia: a random association? Eur Heart J 2020; ehaa254. doi: 10.1093/eurheartj/ehaa254. (Epub ahead of print).

17. Xie Y, Wang X, Yang P, Shuton S: COVID-19 complicated by acute pulmonary embolism. Radiology Cardiothoracic Imaging 2020 Mar 16 10.1148/ryct.20202000067 (Epub ahead of print).

18. Li XY, Du B, Wang YS, et al.: The keypoints in treatment of the critical coronavirus disease 2019 patient. [Article in Chinese.] Zhonghua Jie He Hu Xi Za Zhi 2020 Febr 29 doi: 10.3.3760/ cma.j. cn112147-20200224-00159 (Epub ahead of print).

19. Jin YH, Cai L, Cheng ZS, et al.: A rapid advice guideline for the diagnosis and treatment of 2019 novel coronavirus (2019-nCoV) infected pneumonia (standard version). Mil Med Res 2020; 7, 4.

20. Blank W, Mathis G, Osterwalder J: Kursbuch Notfallsonografie. Stuttgart; Thieme: 2019.

21. Testa A, Soldati G, Copetti R, et al.: Early recognition of the 2009 pandemic influenza $A(H A N+)$ pneumonia by chest ultrasound. Crit Care 2012; 16: R30.

22. Tsung JW, Kessler DO, Saha VP: Prospective application of clinician-performed lung ultrasonography during the 2009 H1N1 influenza A pandemic: distinguishing viral from bacterial pneumonia. Crit Ultrasound J 2012; 4: 16. 
23. Peng S, Zong YM, Shu J, et al.: Dynamic assessment of lung injury by ultrasound in a case with H7N9 influenza. Crit Care 2013; 17: 438.

24. Zhang YK, Li J, Yang JP, Zhan Y, Chen J: Lung ultrasonography for the diagnosis of 11 patients with acute respiratory distress syndrome due to bird flu H7N9 infection. Virology $J$ 2015; 12:176

25. Tsai NW, Ngai CW, Mok KL, Tsung JW: Lung ultrasound imaging in avian influenza A (H7N9) respiratory failure. Crit Ultrasound J 2014; 6: 6.

26. Peng QY, Wang XT, Zhang LN: Findings of lung ultrasonography of novel corona virus pneumonia during the 2019-2020 epidemic. Int Care Med 2020; 1-2. doi: 10.1007/s00134-02005996-6 (Epub ahead of print).

27. Buonosenso D, Piano A, Raffaelli F, Bonada N, De Gaetano K: Point-of-care lung ultrasound findings in novel coronavirus disease-19 pneumoniae: a case report and potential applications during COVID-19 outbreak. Eur Re Med Pharmacological Sci 2020; 24: 2276-2280

28. Poggiali E, Dacrema A, Bastoni D, et al.: Can lung ultrasound help critical care cliniciansin the early diagnosis of novel coronavirus (COVID-19) pneumonia? Radiology 2020; 200847. doi: 10.1148/radiol.2020200847 (Epub ahead of print).

29. Thomas A, Haljan G, Mitra: Lung ultrasound findings in a 64year old woman with COVID-19. CMAJ 2020; cmaj.200414. doi: 10.1503/cmaj.200414 (Epub ahead of print).

30. Huang Y, Wang, Liu Y, et al.: A preliminary study on the ultrasonic manifestation of peripulmonary lesions of non-critical novel coronavirus pneumonia (COVID-10). https://papers.ssrn. com/sol3/papers.cfm?abstract_id=3544750; letzter Zugriff: 04.04.2020.

31. Vertrugno L, Bove T, Orso D, et al.: Our Italian experience using ultrasound for identification, grading and serial follow-up of severity of lung involvement for management for patients with COVID-19. Echocardiography 2020; 10.111/ecjo.14664 (Epub ahead of print).

32. Soldati G, Smargiassi A, Inchingola R, et al.: Is there a role for lung ultrasound during the COVID-19 pandemic? J Ultrasound Med 2020; 10.1002/jum.15284 (Epub ahead of print).
33. Soldati G, Smargiassi A, Inchingola R, et al.: Proposal for international standardization of the use of lung ultrasound for COVID-19 patients; a simple, quantitative, reproducible method. J Ultrasound Med 2020; 10.1002/jum.15285 (Epub ahead of print).

34. Stock K: Lungenultraschall-Protokoll. Klinikum rechts der Isar, Technische Universität München. 2020. unter www.sgum.ch.

35. Stone M: COVID-19 lung ultrasound triage. Butterflynetwork. com/covid-19; letzter Zugriff: 15.04.2020.

36. Mathis G: Triple ultrasound in diagnosis of thromboembolism: EC Pulmonology and Respiratory Medicine 8.2.(2019): 162-168.

37. Kline JA, Garett JS, Sarmineto EJ, Strachan CC, Courtney DM: Over-testing for suspected pulmonary embolism in American emergency departments. The continuing epidemic. Circulation 2020; 13: e005753. doi: 10.1161/CIRCOUTCOMES. 119.005753.

38. American College of Emergency Physicians: ACEP Guidelines on COVD-19: Ultrasound machine and transducer cleaning. 2020 March 31. https://www.acep.org/globalassets/newpdfs/policy-statements/guideline-on-covid-19--ultra sound-machine-and-transducer-cleaning.pdf; letzter Zugriff: 15.04.2020.

\section{Dank}

Ich danke Prof. Gebhard Mathis, PD Dr. Konrad Stock, Dr. Wolfgang Heinz und Dr. Wolfgang Blank für Anregungen und die kritische Durchsicht des Manuskripts.

Manuskript akzeptiert: 13.04.2020

\section{Prof. em. Dr. Joseph Osterwalder, FESEM}

Polipraxis Permanence St. Gallen

Kornhausstrasse 3

9000 St. Gallen

jo@j-osterwalder.ch 